\title{
Controle glicêmico de pacientes diabéticos tipo 2 nos serviços público e privado de Saúde
}

\author{
Glycemic control in type 2 diabetic patients in \\ public and private Healthcare service
}

Daniel Panarotto', Henrique de Araújo Vianna Träsel' ${ }^{1}$, Michele Salibe de Oliveira' ${ }^{1}$, Lívia Brancher Gravina' ${ }^{1}$ Alisson Roberto Teles ${ }^{1}$

\section{RESUMO}

Objetivo: Comparar o tratamento de pacientes diabéticos tipo 2 provenientes de uma clínica privada aos pacientes atendidos no sistema público de Saúde. Métodos: Foi desenhado um estudo de coorte retrospectivo, incluindo 80 pacientes atendidos no Ambulatório Central da Universidade de Caxias do Sul (AMCE), vinculado ao Sistema Único de Saúde, e 277 pacientes atendidos em uma clínica privada, que foram consultados nestes serviços no período entre maio de 2001 e outubro de 2007. Resultados: Os pacientes atendidos no AMCE mostraram pior controle metabólico, embora somente os valores de A1c e colesterol total tenham alcançado significância estatística. Ambos os grupos apresentaram melhora de quase todos os parâmetros metabólicos. $\mathrm{O}$ uso de insulina no final do acompanhamento $(B=4,66 ;$ IC95\% $=2,18-9,89 ; p<0,001)$ e a $A 1 c$ inicial $(B=$ $1,42 ; \mathrm{IC} 95 \%=1,16-1,74 ; p=0,001)$ foram determinantes de pior controle glicêmico. A frequência de consultas foi determinante de melhor controle $(B=0,72 ; I C 95 \%=0,55-0,93 ; p=0,01)$. Conclusões: As variáveis como a A1c inicial e a frequência de consultas, que podem ser consideradas como indicadores do acesso dos pacientes ao sistema de saúde, têm maior impacto no controle do diabetes do que o local no qual os pacientes são tratados. Arq Bras Endocrinol Metab. 2009;53(6):733-40

Descritores

Diabetes melito tipo 2; hemoglobina glicosilada; Sistema Único de Saúde; saúde suplementar

\section{ABSTRACT}

Objective: To compare the treatment of type 2 diabetic patients from a private clinic with those of a public health service. Methods: It was designed a retrospective cohort study, including 80 patients attended at the Central Clinic of Universidade de Caxias do Sul (AMCE), which is related to the Single Health System, and 277 patients attended at a private clinic, whom consult between May 2001 and October 2007. Results: Patients attended at AMCE showed a worse metabolic control, although only the values of $A 1 \mathrm{c}$ and total cholesterol have reached statistical significance. Both groups had an improvement in almost all the metabolic parameters. The use of insulin at the end of follow-up $(B=4,66 ; C 195 \%=2,18-9,89 ; p<0,001)$ and initial $A 1 c(B=1,42$; $\mathrm{Cl} 95 \%=1,16-1,74 ; \mathrm{p}=0,001)$ were determinant of a worse glycemic control. The frequency of visits was determinant of a better control $(B=0,72 ; C l 95 \%=0,55-0,93 ; p=0,01)$. Conclusions: The variables such as the initial $\mathrm{A} 1 \mathrm{c}$ and the frequency of visits, which may be considered as indicators of patients' access to the heath system, have greater impact on the control of diabetes than the place where the patients are treated. Arq Bras Endocrinol Metab. 2009;53(6):733-40

\section{Keywords}

Diabetes mellitus, type 2; hemoglobin glycosylated; Single Health System; supplemental health
${ }^{1}$ Faculdade de Medicina, Universidade de Caxias do Sul (UCS), Rio Grande do Sul, RS, Brasil
Correspondência para:

Daniel Panarotto

UCS, Laboratório de Fisiologia, bloco S, sala 514

Rua Francisco Getúlio Vargas, 1.130 - Petrópolis

95010-550 - Caxias do Sul, RS, Brasil dpanarot@ucs.br

Recebido em 2/Fev/2009 Aceito em 3/Abr/2009

\section{INTRODUÇÃO}

$\mathrm{O}$ diabetes melito tipo 2 (DMT2) acometia $4 \%$ da população adulta mundial no ano de 1995 e esti- ma-se que, em 2025, atingirá cerca de $5,4 \%$ das pessoas (1). Segundo um levantamento epidemiológico realizado em vários Estados brasileiros em 1988, a prevalência 
de diabetes melito (DM) varia entre $5,2 \%$ e $9,7 \%$ entre pessoas de 30 a 69 anos, sendo as regiões Sul e Sudeste as que apresentam maiores taxas da doença (2). Desde então, nenhum grande estudo semelhante foi realizado no Brasil, mas há indícios de que a prevalência de DM também esteja aumentando em nosso país (1).

O DM gera altos custos para os sistemas de saúde. A dimensão deste ônus varia de acordo a prevalência local da doença e a complexidade do tratamento disponível (3). O estudo "The cost of diabetes in Europe - type 2" (CODE-2), que analisou os custos totais referentes ao DMT2 em oito países europeus, identificou que o custo principal para essa doença não é relativo à doença em si ou ao seu tratamento, e sim às complicações causadas por ela (4). No Brasil, no entanto, não há estudos que analisem os custos diretos e indiretos relacionados ao DM.

O controle adequado da glicemia, dos lipídios plasmáticos e da pressão arterial de pacientes com DMT2 retarda ou previne o aparecimento das complicações micro e macrovasculares relacionadas à doença (5-8). Essas evidências motivaram o estabelecimento de metas para o controle desses parâmetros. Acredita-se que a obtenção dessas metas é menos eficaz no sistema de Saúde Pública comparativamente ao sistema de saúde suplementar (9). Entretanto, não existem estudos que tenham avaliado essa importante questão.

Dessa forma, o presente estudo teve como objetivos comparar o tratamento de pacientes diabéticos tipo 2 provenientes de uma clínica privada com os pacientes atendidos no sistema público de Saúde, bem como avaliar os possíveis fatores relacionados ao alcance das metas preconizadas para o controle glicêmico.

\section{MÉTODOS}

Este foi um estudo de coorte, retrospectivo, constituído por uma amostra de pacientes com DMT2, atendidos no Serviço de Endocrinologia do Ambulatório Central da Universidade de Caxias do Sul (AMCE), o qual está vinculado exclusivamente ao Sistema Único de Saúde (SUS), e de uma amostra de pacientes com diagnóstico de DMT2 provenientes de um consultório privado. No consultório são atendidos pacientes com planos de saúde e particulares (os quais totalizam menos de $5 \%$ das consultas). O tempo médio dedicado ao paciente nessas consultas é de 20 a 30 minutos.

Em ambos os serviços, os pacientes foram diagnosticados conforme as recomendações da American Diabetes Association (ADA) (10). O mesmo médico endo- crinologista coordenador deste estudo assistiu tanto os pacientes do AMCE quanto os da clínica privada.

A dinâmica da coleta de dados dos pacientes do AMCE (11) ocorreu da seguinte maneira: os pacientes foram vistos por uma equipe multidisciplinar, composta por uma enfermeira, um médico endocrinologista, acadêmicos de Medicina e uma nutricionista. O tempo médio dedicado ao paciente nestas consultas foi de 30 a 40 minutos. Os dados da anamnese e do exame físico foram anotados em um protocolo padrão, criado para uniformizar o registro das informações obtidas na consulta. No consultório privado, até o ano de 2004 , as consultas eram registradas em prontuários convencionais. A partir dessa data, adotou-se o registro eletrônico das consultas, utilizando-se o programa D-Clinisoft Consultório ${ }^{\circledR}$, versão 4.9. Dessa forma, foram revisadas as informações contidas nestes dois meios de registro. Todas as informações obtidas foram organizadas em uma planilha do programa Microsoft Excel ${ }^{\circledR}$, versão 2003. Os pacientes que apresentavam tempo de acompanhamento menor que seis meses foram excluídos da análise.

Obtiveram-se os dados das consultas realizadas entre agosto de 2001 e outubro de 2007 no AMCE, e entre maio de 2001 e outubro de 2007 no consultório privado. O médico endocrinologista seguiu, em ambos os grupos, a mesma rotina de atendimento e solicitação de exames complementares. Dessa forma, os seguintes dados foram coletados: gênero; data de nascimento, ano de diagnóstico de DM e data das consultas (esses dados foram utilizados para o cálculo da idade ao diagnóstico do DM, da duração do DM, e da idade do paciente); presença de comorbidades; altura; peso; índice de massa corpórea (IMC); circunferência abdominal; pressão arterial sistólica (PAS) e diastólica (PAD); glicemia em jejum; glicemia pós-prandial; hemoglobina glicada (Alc); colesterol total; colesterol HDL; colesterol LDL e triglicerídeos; medicamentos em uso.

\section{Definições}

O início do acompanhamento foi definido como a primeira consulta do paciente em ambos os grupos. Todos os dados de exame físico, medicamentos em uso e comorbidades utilizados neste estudo foram coletados na primeira consulta. Para os pacientes que, na primeira consulta, traziam exames laboratoriais realizados nos três meses anteriores à data do primeiro atendimento, estes foram utilizados como exames iniciais. Caso o paciente não tivesse exames, esses foram solicitados e os resultados, 
anotados na consulta subsequente (segunda consulta) e considerados como exames iniciais para fins de análise. O fim do acompanhamento foi definido como a última consulta do paciente no período supracitado. Nesta, novamente obtiveram-se os dados referentes ao exame físico, medicamentos em uso e exames laboratoriais.

Foi utilizado como referência o atual consenso da ADA (12) para definir o controle metabólico desejado, que consiste em glicemia de jejum $\geq 70 \mathrm{mg} / \mathrm{dL}$ e $\leq 130 \mathrm{mg} / \mathrm{dL}$, glicemia pós-prandial $<180 \mathrm{mg} / \mathrm{dL}, \mathrm{Alc}<7 \%$, colesterol total $<200 \mathrm{mg} / \mathrm{dL}$, colesterol-HDL $>40 \mathrm{mg} / \mathrm{dL}$ em homens e $>50 \mathrm{mg} / \mathrm{dL}$ em mulheres, colesterol-LDL $<100$ $\mathrm{mg} / \mathrm{dL}$, triglicerídeos $<150 \mathrm{mg} / \mathrm{dL}, \mathrm{PAS}<130 \mathrm{mmHg}$ e diastólica $<80 \mathrm{mmHg}$ e IMC $<30 \mathrm{~kg} / \mathrm{m}^{2}$.

Os pacientes provenientes do AMCE tiveram seus exames realizados por meio dos métodos padronizados pelo laboratório local. Neste, o método de análise da Alc é a Cromatografia Líquida de Alta Eficiência (do inglês High Performance Liquid Chromatography, HPLC), o qual é certificado pelo Programa de Padronização Nacional da Glico-hemoglobina (NGSP) (13). Os pacientes da clínica privada trouxeram exames realizados nos laboratórios da região, os quais, em sua maioria, também utilizam o mesmo método (13). Excluíram-se das análises os exames realizados por métodos não padronizados pelo NGSP.

\section{Análise estatística}

As análises estatísticas foram conduzidas com o programa Statistical Package for the Social Sciences ${ }^{\circledR}$ (SPSS) para Windows (SPSS Inc., Chicago, IL, USA). As variáveis categóricas foram apresentadas como proporções. As variáveis contínuas foram submetidas ao teste de aderência de Kolmogorov-Smirnov para verificação de normalidade, sendo as variáveis com distribuição normal apresentadas como média e desvio-padrão e as sem distribuição normal, como mediana e intervalo interquartil. Para análise entre AMCE e clínica privada, foram utilizados os testes $t$ de Student e Mann-Whitney para variáveis contínuas com distribuição normal e não normal, respectivamente, e o teste $\chi^{2}$ para variáveis categóricas. Realizou-se análise pareada entre o início e fim do tratamento, utilizando-se os testes $t$ pareado e Wilcoxon para variáveis contínuas com distribuição paramétrica e não paramétrica, respectivamente, e o teste de McNemar para variáveis categóricas.

Para avaliação das variáveis associadas ao mau controle glicêmico, foi utilizado como ponto de corte o valor de $\mathrm{Alc}<7 \%$. Inicialmente, realizou-se uma análise univariada, utilizando-se o teste $t$ em variáveis contínuas com distribuição normal, o teste de Mann-Whitney em variáveis sem distribuição gaussiana, e o teste $\chi^{2}$ para variáveis categóricas. As variáveis com $\mathrm{p} \leq 0,15$ na análise univariada foram posteriormente analisadas em regressão logística. Utilizou-se o método de entrada por blocos (block entry) para seleção do modelo final da regressão.

\section{Considerações éticas}

Este estudo foi conduzido de acordo com a Declaração de Helsinque.

\section{RESULTADOS}

Analisaram-se os dados de 357 pacientes, 80 provenientes do AMCE e 277 da clínica privada. Algumas características dos pacientes estudados são apresentadas na tabela 1 . Os grupos são semelhantes quanto à idade ao início do acompanhamento e à idade ao diagnóstico de DM. Em relação às comorbidades, é notável a presença de pacientes hipertensos, que totalizam, no geral, $75 \%$, correspondendo a $90 \%$ dos pacientes atendidos no AMCE e a $70,7 \%$ dos pacientes da clínica privada $(\mathrm{p}=0,9)$. A maioria dos pacientes encontrava-se com sobrepeso ou obesidade $78,6 \%$ no AMCE versus $73,1 \%$ na clínica privada), sendo a porcentagem de pacientes com obesidade grau III diferente entre os grupos $(\mathrm{p}=0,01)$.

Na tabela 2, é descrita a comparação entre diversos parâmetros metabólicos dos pacientes, no início e no final do período de acompanhamento. Pôde-se observar que, no início do acompanhamento, pacientes tratados no AMCE estavam, em geral, com pior controle metabólico em relação aos pacientes atendidos na clínica privada, embora apenas os valores de Alc tenham alcançado significância estatística. No final do período de acompanhamento, não só essa diferença persistiu, como também foi observada uma diferença nas concentrações de colesterol total, que foi igualmente mais elevada nos pacientes do AMCE.

Durante o acompanhamento, houve melhora de quase todos os parâmetros metabólicos em ambos os grupos (Tabela 2). Essa melhora está associada às mudanças no tratamento dos pacientes, como se pode observar na figura 1. Em resumo, um grande número de pacientes que eram tratados com dieta e exercícios passou a usar metformina, enquanto muitos que utilizavam somente antidiabéticos orais passaram a usar insulina (associada ou não a antidiabéticos orais). 


\begin{tabular}{|c|c|c|c|}
\hline & AMCE $(n=80)$ & Clínica privada $(\mathrm{n}=277$ ) & $p$ \\
\hline Gênero feminino & $57(71,2)$ & $148(53,4)$ & $<0,01$ \\
\hline Idade no início do acompanhamento (em anos) ${ }^{\star *}$ & $56,7 \pm 12,2$ & $57,3 \pm 11,8$ & 0,70 \\
\hline Idade ao diagnóstico de diabetes (em anos) ${ }^{\star \star}$ & $48,9 \pm 12,2$ & $50,0 \pm 11,3$ & 0,42 \\
\hline$N^{\circ}$ de consultas* & $6,0(4,0-8,0)$ & $7,0(5,0-10,0)$ & 0,01 \\
\hline Frequência de consultas (por ano) ${ }^{\star \star}$ & $3,6 \pm 1,15$ & $4,5 \pm 1,8$ & $<0,01$ \\
\hline Duração de diabetes (em anos)* & $7,4(3,9-13,0)$ & $7,2(3,4-12,7)$ & 0,45 \\
\hline Tempo de tratamento (em anos) ${ }^{\star}$ & $1,7(0,94-2,85)$ & $1,75(0,93-3,0)$ & 0,69 \\
\hline \multicolumn{4}{|l|}{ Comorbidades } \\
\hline HAS & $72(90,0)$ & $196(70,7)$ & 0,90 \\
\hline Tabagismo & $15(18,7)$ & $34(12,2)$ & 0,13 \\
\hline Sobrepeso & $29(36,2)$ & $95(34,2)$ & 0,74 \\
\hline Obesidade grau I & $20(25,0)$ & $71(25,6)$ & 0,90 \\
\hline Obesidade grau II & $7(8,7)$ & $30(10,8)$ & 0,59 \\
\hline Obesidade grau III & $7(8,7)$ & $7(2,5)$ & 0,01 \\
\hline
\end{tabular}

Valores dados em n (\%). * Valores dados em mediana (P25-P75). ** Valores dados em média \pm DP.

Tabela 2. Comparação das características laboratoriais e do exame físico entre pacientes diabéticos tipo 2 do AMCE e da clínica privada $(n=357)$

\begin{tabular}{|c|c|c|c|c|c|c|}
\hline & \multicolumn{2}{|c|}{ Início } & \multicolumn{4}{|c|}{ Fim } \\
\hline & AMCE & Clínica privada & $\mathbf{p}$ & AMCE & Clínica privada & $\mathbf{p}$ \\
\hline Glicemia em jejum (mg/dL) & $191,5 \pm 88,5$ & $180,4 \pm 75,1$ & 0,47 & $147,9 \pm 78,5 \neq$ & $135,0 \pm 48,6 \neq$ & 0,89 \\
\hline Glicemia pós-prandial (mg/dL) & $244,9 \pm 127,5$ & $214,8 \pm 103,3$ & 0,21 & $184,4 \pm 82,0 \neq$ & $170,2 \pm 75,8 \ddagger$ & 0,22 \\
\hline Hemoglobina glicada (\%) & $9,7 \pm 2,3$ & $8,3 \pm 2,3$ & $<0,01$ & $8,3 \pm 2,0 \neq$ & $7,5 \pm 1,8 \neq$ & $<0,01$ \\
\hline Colesterol total (mg/dL) & $205,1 \pm 48,5$ & $205,8 \pm 44,1$ & 0,91 & $188,7 \pm 42,9 \ddagger$ & $172,1 \pm 39,0 \neq$ & $<0,01$ \\
\hline $\mathrm{HDL}-\mathrm{C}(\mathrm{mg} / \mathrm{dL})$ & $41,9 \pm 12,9$ & $42,6 \pm 11,8$ & 0,62 & $44,7 \pm 14,9$ & $42,9 \pm 11,6$ & 0,57 \\
\hline $\mathrm{LDL}-\mathrm{C}(\mathrm{mg} / \mathrm{dL})$ & $126,0 \pm 42,1$ & $122,2 \pm 44,2$ & 0,54 & $106,4 \pm 31,2 \ddagger$ & $98,6 \pm 35,1 \neq$ & 0,15 \\
\hline Triglicerídeos $(\mathrm{mg} / \mathrm{dL})^{*}$ & $178,0(111,0-233,0)$ & $169,5(111,7-243,7)$ & 0,84 & $141,0(122,0-217,0)$ & $146,0(101,5-192,5) \ddagger$ & 0,32 \\
\hline IMC $\left(\mathrm{kg} / \mathrm{m}^{2}\right)$ & $30,3 \pm 6,0$ & $29,7 \pm 5,5$ & 0,37 & $30,5 \pm 5,7$ & $29,5 \pm 5,6$ & 0,20 \\
\hline PAS (mmHg) & $138,8 \pm 18,4$ & $142,3 \pm 26,6$ & 0,58 & $129,5 \pm 19,9 \ddagger$ & $133,3 \pm 22,8 \ddagger$ & 0,24 \\
\hline PAD (mmHg) & $85,3 \pm 12,7$ & $83,7 \pm 14,6$ & 0,44 & $80,2 \pm 11,4 \ddagger$ & $77,1 \pm 10,5 \ddagger$ & 0,10 \\
\hline
\end{tabular}

Valores dados em média \pm DP. * Valores dados em mediana (P25-P75). $¥$ Comparação dos valores entre início e fim do tratamento $(p \leq 0,05)$.

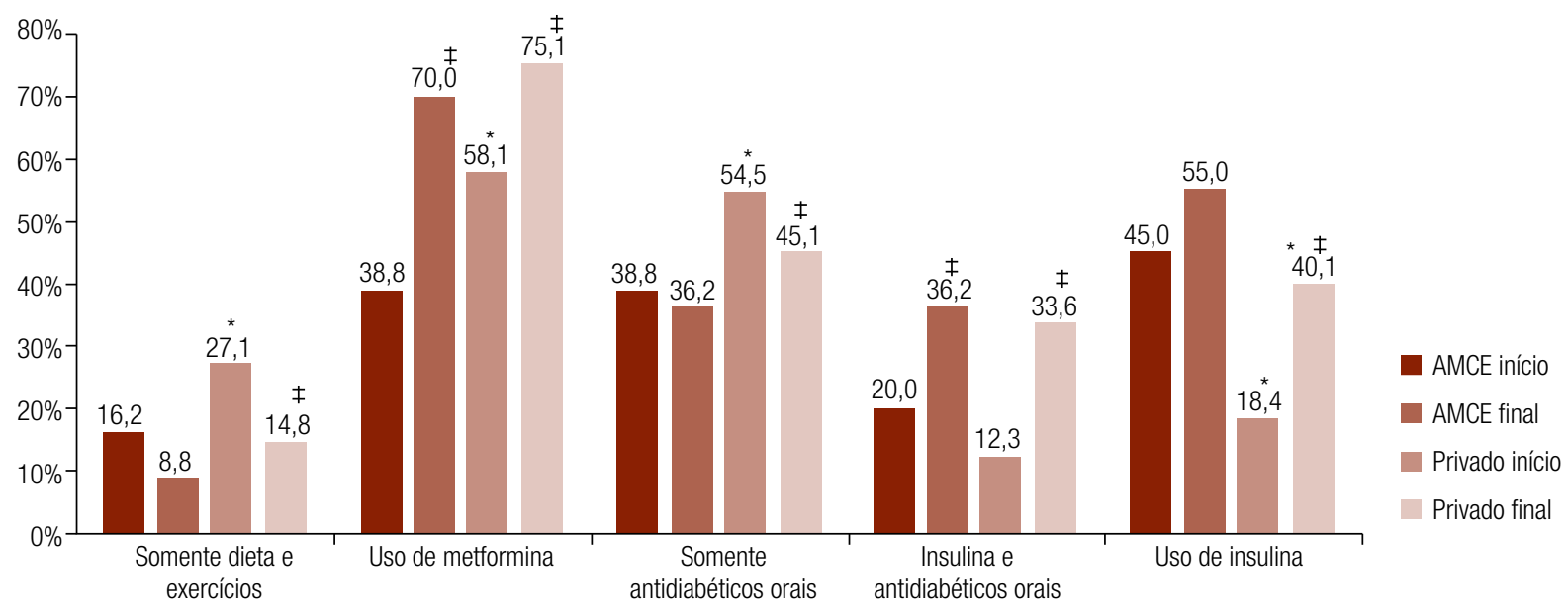

Todos os valores estão representados em porcentagem (\%). * $p \leq 0,05$ para comparação entre AMCE e privado. $\ddagger p \leq 0,05$ para comparação entre início e fim de cada grupo.

Figura 1. Medicamentos no início e no final do acompanhamento nos pacientes diabéticos tipo 2 do AMCE e da clínica privada. 
$\mathrm{Na}$ figura 2, foi observado que uma minoria dos pacientes estava dentro das metas de tratamento recomendadas no início do acompanhamento. A despeito da melhora observada, a maioria dos pacientes ainda estava fora desses alvos no fim do acompanhamento.

No final do período de acompanhamento, os fatores que influenciaram no controle glicêmico, definido com Alc $>7 \%$, foram estudados por meio de uma análise de regressão logística. Os resultados dessa análise mostraram que o uso de insulina no final do acompanhamento $(\mathrm{B}=4,66 ; \mathrm{IC} 95 \%=2,18-9,89 ; \mathrm{p}<0,001)$ e a Alc inicial $(\mathrm{B}=1,42 ; \mathrm{IC} 95 \%=1,16-1,74 ; \mathrm{p}=0,001)$ foram determinantes de pior controle glicêmico, enquanto a frequência de consultas foi determinante de melhor controle $(\mathrm{B}=0,72 ; \mathrm{IC} 95 \%=0,55-0,93 ; \mathrm{p}=0,01)$.

\section{DISCUSSÃO}

Neste estudo, comparou-se o tratamento de pacientes diabéticos tipo 2 em uma amostra proveniente de uma clínica privada com outra retirada de um ambulatório servido pelo SUS.

Foi possível observar que os grupos são semelhantes quanto a uma série de características, excetuando-se a porcentagem de mulheres atendidas no AMCE, a frequência e o número de consultas, além da porcentagem de indivíduos com obesidade grau III. O primeiro achado constitui provavelmente um viés de seleção, já que outros estudos demonstraram que a prevalência de DM é semelhante em homens e mulheres (2).
Em nosso estudo, a minoria dos pacientes atingiu os alvos de Alc, colesterol LDL, triglicerídeos e pressão arterial preconizados pela ADA. Além disso, se forem utilizados pontos de corte menores para Alc, como propõem, por exemplo, a European Association for the Study of Diabetes (EASD) (14), a International Diabetes Federation (IDF) (15) e a American Association of Clinical Endocrinologists (AACE) (16), a porcentagem de pacientes que se encontram com controle adequado é ainda menor (dados não apresentados). Esses dados corroboram os resultados de outros estudos, nos quais as metas de tratamento propostas pela ADA foram alcançadas por uma minoria dos pacientes $(17,18)$.

Porém, mesmo que as metas de controle metabólico não tenham sido atingidas pela maioria dos pacientes, é esperado que a melhora dos parâmetros metabólicos ocorrida do início para o fim do acompanhamento tenha um impacto significativo na redução das complicações crônicas do DM, incluindo redução na mortalidade $(7,19,20)$. No estudo United Kingdom Prospective Diabetes Study Group (UKPDS), por exemplo, cada $1 \%$ na redução da Alc foi associado a um decréscimo estimado de $37 \%$ no risco de desenvolvimento de complicações microvasculares, $14 \%$ no risco de infarto do miocárdio, $12 \%$ no risco de doença vascular periférica e $21 \%$ na mortalidade relacionada ao DM (20).

Cabe salientar que nem todos os pacientes têm indicação para realização do controle muito intensivo da glicemia. Estudos recentes demonstraram que essa estratégia de tratamento não reduziu a mortalidade e a inci-

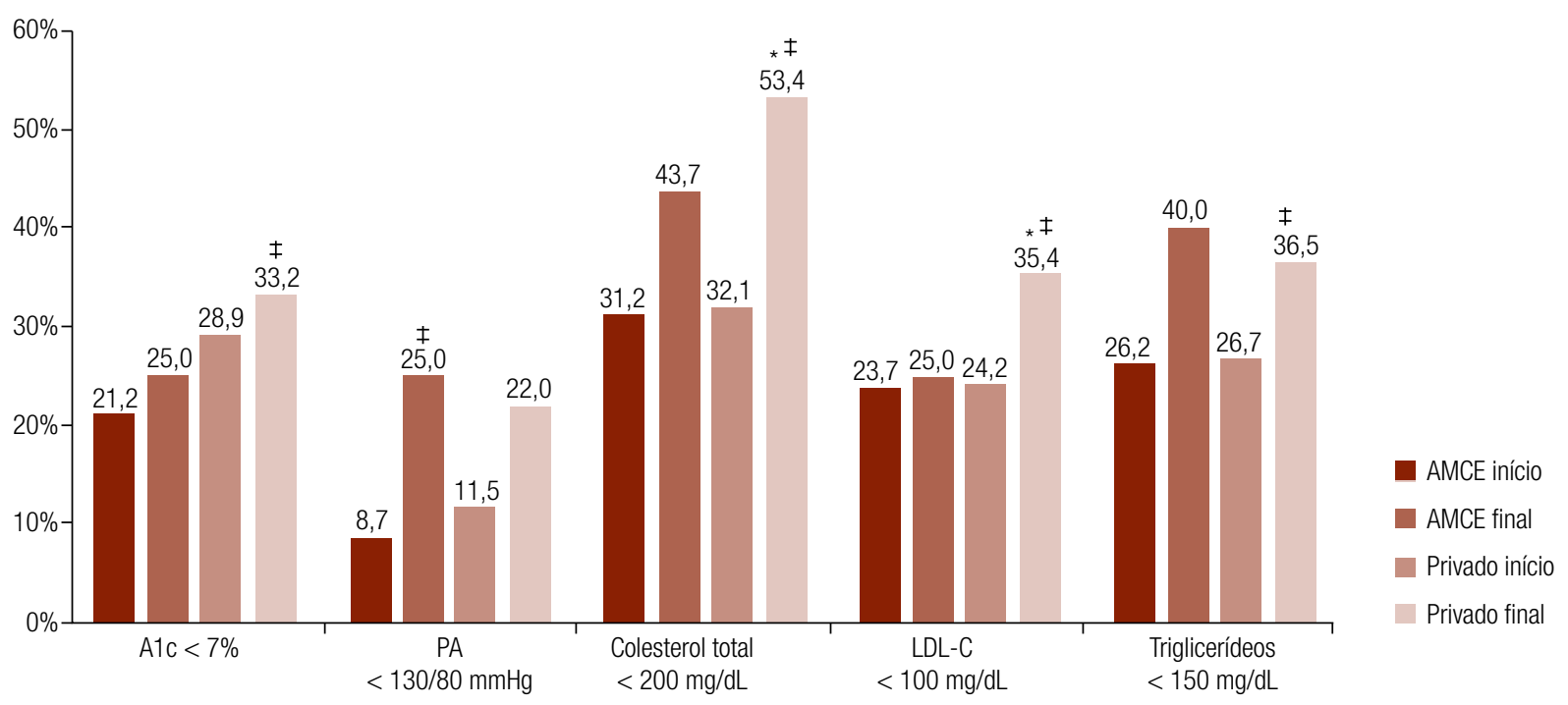

Todos os valores estão representados em porcentagem (\%). ${ }^{*} p \leq 0,05$ para comparação entre AMCE e privado. $\ddagger p \leq 0,05$ para comparação entre início e fim de cada grupo.

Figura 2. Controle metabólico dos pacientes diabéticos tipo 2 do AMCE e da clínica privada no início e no final do acompanhamento. 
dência de desfechos cardiovasculares $(21,22)$, enquanto outros demonstraram até um aumento da mortalidade dos pacientes diabéticos e não diabéticos submetidos a uma diminuição agressiva da glicemia $(23,24)$.

Portanto, pacientes que apresentam expectativa de vida diminuída, complicações em estágio clínico avançado, múltiplos episódios de hipoglicemia severa ou dificuldade de reconhecimento de hipoglicemia podem ter alvos de tratamento mais elevados (5).

A obtenção da melhora nos parâmetros glicêmicos dos pacientes deste estudo foi alcançada por meio de uma mudança no tratamento. Primeiro, pacientes somente em dieta passaram a receber metformina, uma conduta que respeita as recomendações vigentes para o tratamento do diabetes $(25,26)$. Segundo, pacientes em uso de antidiabéticos orais, isoladamente ou em combinação, que não estavam bem controlados, receberam insulina.

No início do acompanhamento, observou-se que a Alc e, no final do tratamento, o uso de insulina foram variáveis independentes associadas ao controle glicêmico inadequado. Por outro lado, a frequência de consultas foi um fator protetor. Foram interpretados esses dados da seguinte maneira: usuários de insulina, em geral, têm maior duração de DM (mediana (P25-P75) em anos: $10,6(6,4-16,4)$ versus $5,2(2,6-9,3) ; \mathrm{p}<0,01)$ e estão mais descompensados (Alc) tanto no início (média \pm DP em $\%: 9,7 \pm 2,4$ versus $7,6 \pm 1,8 ; \mathrm{p}<0,01)$ quanto no fim do acompanhamento (média \pm DP em \%: 8,8 \pm $2,2$ versus $7,02 \pm 1,2 ; \mathrm{p}<0,01)$ que pacientes que não estão usando insulina. Dessa forma, mesmo que os pacientes que iniciaram o uso de insulina tenham obtido significativa melhora do controle glicêmico, eles ainda persistem acima dos alvos de tratamento.

Esse tipo de resultado já foi observado em outro estudo publicado pelo nosso grupo (11), assim como em dois trabalhos recentes realizados na Alemanha $(27,28)$. Yurgin e cols. observaram que pacientes tratados exclusivamente com insulina ou com antidiabéticos orais e insulina foram menos propensos a alcançar a meta de Alc em relação aos pacientes que fizeram uso de metformina ou sulfonilureia isoladamente ou em combinação (28). Da mesma forma, Huppertz e cols. identificaram que pacientes com menor duração do diabetes e que, portanto, não necessitam de nenhuma intervenção farmacológica têm melhor controle metabólico do que aqueles em uso de insulina ou combinação de antidiabéticos orais (27). No estudo UKPDS 35 também foi constatada a relação entre a dificuldade em obter e manter a concentração satisfatória da Alc em pacientes com DMT2 - particularmente nos pacientes com alto percentual de Alc na época do diagnóstico (20).

$\mathrm{O}$ mesmo raciocínio vale para considerar o valor prognóstico da Alc inicial como fator explicativo da Alc final. É importante observar que essa variável se torna mais importante no nosso modelo de análise multivariada do que o local de tratamento (AMCE versus clínica privada), já que esta última perde a significância estatística face à entrada da Alc inicial no modelo.

Além disso, nosso modelo de regressão identificou que quanto maior a frequência de consultas, melhor o controle glicêmico no final do acompanhamento. Como a melhora do controle glicêmico foi semelhante entre os grupos, entre o início e o final do acompanhamento, entendeu-se que a frequência de consultas pode ser um indicador do acesso do paciente ao sistema de saúde.

Nosso estudo mostra que usuários do SUS têm um pior controle glicêmico no início do acompanhamento em relação aos usuários da saúde suplementar. Esse dado pode ser explicado pelo fato de que usuários da saúde suplementar têm mais acesso a atendimento especializado do que os beneficiários do SUS, chegando mais bem compensados ao primeiro atendimento médico. Por outro lado, pacientes atendidos no sistema de saúde pública, uma vez que têm acesso ao atendimento especializado, obtêm melhora tão boa ou mesmo melhor do controle glicêmico do que pacientes atendidos em uma clínica privada. Essa assertiva é corroborada pelo dado de que a diferença entre Alc inicial e final nos usuários do SUS é maior do que nos da clínica privada ( $1,5 \%$ versus $0,8 \%)$.

É digno de nota o fato de que o tempo aproximado de espera para uma consulta na clínica privada é de aproximadamente 30 dias, enquanto, no AMCE, esse tempo é, em média, de seis a oito meses.

Estudos anteriores mostraram que pacientes com DMT2 atendidos no sistema privado de saúde apresentaram um controle pobre, mas sugerem que resultados piores podem ser esperados entre os usuários do sistema público de saúde (9).

Os motivos usados para explicar essa suposta diferença são, entre outros, o menor acesso a medicamentos e o pior atendimento médico entre os pacientes do SUS. O que se observou, no entanto, foi a situação oposta. Pacientes atendidos no AMCE, por exemplo, têm acesso a aconselhamento nutricional. Além disso, o tempo de consulta é certamente maior no ambiente acadêmico desse ambulatório, e os pacientes provavel- 
mente recebem orientações mais detalhadas dos acadêmicos de Medicina que conduzem a consulta. Todas essas facilidades, por maior que seja o esforço do médico, são menos frequentes na clínica privada, onde o atendimento multidisciplinar é mais difícil de ser obtido.

Portanto, os resultados aqui apresentados sugerem que o tratamento oferecido pelo SUS ao paciente com DM pode ser tão eficaz quanto o oferecido pelo sistema de saúde suplementar, sendo o controle glicêmico prejudicado, nos primeiros, provavelmente, pela dificuldade de acesso do usuário ao atendimento.

Nosso estudo tem algumas limitações. Primeiro, por tratar-se de uma amostra não aleatória, há a possibilidade de viés de seleção. A amostra utilizada em nosso estudo, tanto a proveniente do AMCE quanto aquela advinda da clínica privada, pode não representar as populações de diabéticos as quais pertencem, tanto com relação às características socioeconômicas quanto às biológicas. Portanto, a inferência de que o tratamento proporcionado pelo SUS é equivalente ao proporcionado pela saúde suplementar pode não ser aplicável ao conjunto total de diabéticos tipo 2 do Brasil.

Segundo, por não ser um estudo randomizado, muitas variáveis - incluindo aquelas que não foram avaliadas, como condição socioeconômica, escolaridade, entre outras - diferem entre os grupos e podem constituir um viés na interpretação de resultados.

Terceiro, por se tratar de uma revisão de prontuários, não se tem controle sobre a coleta de informações. Por isso, mesmo que se tenha uma sistemática de coleta de dados em ambos os serviços, não se pode descartar algumas imprecisões nas informações obtidas assim como a falta de alguns dados, o que pode ter limitado o tamanho de nossa amostra.

Portanto, outros estudos com delineamento prospectivo e com amostragem representativa das respectivas populações são necessários para confirmar nossos dados. Apesar dessas limitações, nosso estudo é o primeiro que se propôs a comparar o atendimento em pacientes diabéticos tipo 2 provenientes de serviços de saúde público e privado no Brasil, e os resultados aqui apresentados levantam questões que merecem consideração futura.

Em conclusão, nosso estudo demonstrou que o sistema de saúde no qual o indivíduo é tratado não é um determinante do controle glicêmico em pacientes com DMT2. Por outro lado, variáreis relacionadas ao acesso do paciente às consultas, como o controle inicial do diabetes e a frequência de consultas, podem ser fatores mais importantes. Dessa forma, medidas que visam a uma educação continuada em saúde no nível primário e uma otimização do acesso ao serviço especializado possivelmente terão uma repercussão no controle dos parâmetros metabólicos dos pacientes com DMT2 e, desse modo, na redução da morbimortalidade associada a essa doença de tão alto impacto na saúde pública brasileira.

Declaração: os autores declaram não haver conflitos de interesse científico neste estudo.

\section{REFERÊNCIAS}

1. King H, Aubert RE, Herman WH. Global burden of diabetes, 19952025: prevalence, numerical estimates, and projections. Diabetes Care. 1998;21:1414-31.

2. Malerbi DA, Franco LJ. Multicenter study of the prevalence of diabetes mellitus and impaired glucose tolerance in the urban Brazilian population aged 30-69 yr. The Brazilian Cooperative Group on the Study of Diabetes Prevalence. Diabetes Care. 1992;15(11):1509-16.

3. Brasil. Ministério da Saúde. Secretaria de Atenção Básica. Departamento de Atenção Básica. Diabetes Mellitus. Cadernos de Atenção Básica. Brasília; 2006.

4. Jönsson B, CODE-2 Advisory Board. Revealing the cost of type II diabetes in Europe. Diabetologia. 2002;45:S5-12.

5. [No authors listed]. The effect of intensive treatment of diabetes on the development and progression of long-term complications in insulin-dependent diabetes mellitus. The Diabetes Control and Complications Trial Research Group. N Engl J Med. 1993;329(14):977-86.

6. [No authors listed]. Intensive blood-glucose control with sulphonylureas or insulin compared with conventional treatment and risk of complications in patients with type 2 diabetes (UKPDS 33). United Kingdom Prospective Diabetes Study Group (UKPDS). Lancet. 1998;352(9131):837-53.

7. Gaede $P$, Vedel $P$, Larsen N, Jensen GV, Parving $H H$, Pedersen $O$. Multifactorial intervention and cardiovascular disease in patientes with type 2 diabetes. N Engl J Med. 2003;348(5):383-93.

8. Shichiri M, Kishikawa H, Ohkubo Y, Wake N. Long-term results of the Kumamoto Study on optimal diabetes control in type 2 diabetic patients. Diabetes Care. 2000;23(Suppl 2):B21-9.

9. Lopez Stewart G, Tambascia M, Rosas Guzmán J, Etchegoyen F, Ortega Carrión J, Artemenko S. Control of type 2 diabetes mellitus among general practitioners in private practice in nine countries of Latin America. Rev Panam Salud Publica. 2007;22(1):12-20.

10. [No authors listed]. American Diabetes Association. Clinical Practice Recommendations 2001. Diabetes Care. 2001;24(Suppl 1):S1-133.

11. Panarotto D, Teles AR, Schumacher MV. Fatores associados ao controle glicêmico em pacientes com diabetes tipo 2. Rev Assoc Med Bras. 2008;54(4):314-21.

12. American Diabetes Association. Standards of medical care in diabetes - 2009. Diabetes Care. 2009;32 Suppl 1:S13-61.

13. Panarotto D, Toss AMM, Teles AR. Levantamento dos métodos de análise de hemoglobina glicada utilizados em laboratórios da Serra Gaúcha. Scientia Med. 2005;15(3):137-41.

14. Rydén L, Standl E, Bartnik M, Van den Berghe G, Betteridge J, de Boer $M$, et al. Guidelines on diabetes, pre-diabetes, and cardiovascular diseases: executive summary. The Task Force on Diabetes and Cardiovascular Diseases of the European Society of 
Cardiology (ESC) and of the European Association for the Study of Diabetes (EASD). Eur Heart J. 2007;28(1):88-136.

15. IDF Clinical Guidelines Task Force. Global Guideline for Type 2 Diabetes: recommendations for standard, comprehensive, and minimal care. Diabet Med. 2006;23(6):579-93.

16. Rodbard HW, Blonde L, Braithwaite SS, Brett EM, Cobin RH, Handelsman Y; AACE Diabetes Mellitus Clinical Practice Guidelines Task Force. American Association of Clinical Endocrinologists medical guidelines for clinical practice for the management of diabetes mellitus. Endocr Pract. 2007;Suppl 1:1-68.

17. McFarlane SI, Jacober SJ, Winer N, Kaur J, Castro JP, Wui MA, et al. Control of cardiovascular risk factors in patients with diabetes and hypertension at urban academic medical centers. Diabetes Care. 2002;25(4):718-23.

18. Saydah SH, Fradkin J, Cowie CC. Poor control of risk factors for vascular disease among adults with previously diagnosed diabetes. JAMA. 2004;291(3):335-42.

19. Gaede $P$, Lund-Andersen $H$, Parving $H H$, Pedersen O. Effect of a multifactorial intervention on mortality in type 2 diabetes. $\mathrm{N}$ Engl J Med. 2008;358(6):580-91.

20. Stratton I, Adler A, Neil H, Matthews D, Manley S, Cull C, et al. Association of glycaemia with macrovascular and microvascular complications of type 2 diabetes (UKPDS 35): prospective observational study BMJ. 2000;321(7258):405-12.

21. ADVANCE Collaborative Group, Patel A, MacMahon S, Chalmers $\mathrm{J}$, Neal B, Billot L, et al. Intensive blood glucose control and vascular outcomes in patients with type 2 diabetes. N Engl $\mathrm{J}$ Med. 2008;358(24):2560-72.
22. Duckworth W, Abraira C, Moritz T, Reda D, Emanuele N, Reaven $\mathrm{PD}$, et al. Glucose control and vascular complications in veterans with type 2 diabetes. N Engl J Med. 2009;360(2):129-39.

23. Action to Control Cardiovascular Risk in Diabetes Study Group, Gerstein HC, Miller ME, Byington RP, Goff DC Jr, Bigger JT, et al. Effects of intensive glucose lowering in type 2 diabetes. $N$ Engl $J$ Med. 2008;358(24):2545-59.

24. NICE-SUGAR Study Investigators, Finfer S, Chittock DR, Su SY, Blair D, Foster D, et al. Intensive versus conventional glucose control in critically ill patients. N Engl J Med. 2009;360(13):1283-97.

25. Nathan D, Buse J, Davidson M, Ferrannini E, Holman R, Sherwin $\mathrm{R}$, et al. Management of hyperglycemia in type 2 diabetes: a consensus algorithm for the initiation and adjustment of therapy: update regarding thiazolidinediones: a consensus statement from the American Diabetes Association and the European Association for the Study of Diabetes. Diabetes Care. 2008;31(1):173-5.

26. Nathan D, Buse J, Davidson M, Heine R, Holman R, Sherwin R, et al. Management of hyperglycemia in type 2 diabetes: a consensus algorithm for the initiation and adjustment of therapy. Diabetes Care. 2006;29(8):1963-72.

27. Huppertz E, Pieper L, Klotsche J, Stridde E, Pittrow D, Böhler S, et al. Diabetes mellitus in German primary care: quality of glycaemic control and subpopulations not well controlled - Results of the DETECT Study. Exp Clin Endocrinol Diabetes. 2009;117(1):614. Epub 2008 May 9.

28. Yurgin N, Secnik K, Lage M. Antidiabetic prescriptions and glycemic control in German patients with type 2 diabetes mellitus: a retrospective database study. Clin Ther. 2007;29(2):316-25. 